\title{
Re: Interleukin-11 Attenuates Ifosfamide-Induced Hemorrhagic Cystitis
}

\author{
Jose M. Mota, Gerly A. Brito, Raphael T. Loiola, Fernando Q. Cunha, Ronaldo de A. Ribeiro \\ Departments of Physiology and Pharmacology (JMM, RTL, RAR), and Morphology (GAB), School of \\ Medicine, Federal University of Ceara and Department of Pharmacology FQC), School of Medicine, \\ University of Sao Paulo Ribeirao Preto, Sao Paulo, Brazil
}

Int Braz J Urol, 33: 704-710, 2007

To the Editor:

Ifosfamide (IFS) is a widely used antineoplastic agent, and the occurrence of ifosfamide-induced hemorrhagic cystitis (HC) continues to be a significant problem in spite of adequate uroprotection. Acrolein, the toxic metabolite of IFS, is the main molecule responsible for this side-effect and mesna (2-mercaptoethane sulfonate) is the most commonly used preventative agent. Mesna binds acrolein and prevent its direct contact with uroepithelium. Current knowledge provides information about the pathophysiologic mechanism of HC. Several transcription factors and cytokines, free radicals and non-radical reactive molecules, as well as Poly (ADP-ribose) polymerase (PARP) activation are now known to take part in its pathogenesis., Whether or not it follows chemotherapy (CP), HC is an inflammatory process. Thus, many cytokines such as tumor necrosis factor (TNF), the interleukin (IL) family, transcription factors such as nuclear factor- $\kappa \mathrm{B}(\mathrm{NF}-\kappa \mathrm{B})$ and activator protein-1 (AP-1) also play a role in its pathogenesis. When these molecular factors are taken into account, pathogenesis of CP-induced bladder toxicity can be summarized in three steps: (1) acrolein rapidly enters the uroepithelial cells; (2) it then activates intracellular reactive oxygen species and nitric oxide production (directly or through NF$\kappa \mathrm{B}$ and $\mathrm{AP}-1)$ leading to peroxynitrite production; (3) finally, the increased peroxynitrite level damages lipids (lipid peroxidation), proteins (protein oxidation) and DNA (strand breaks) leading to activation of PARP, a DNA repair enzyme. DNA damage causes PARP overactivation, resulting in the depletion of oxidized nicotinamide-adenine dinucleotide and ATP, and consequently in necrotic cell death. For more effective prevention against HC, all pathophysiologic mechanisms must be taken into consideration.

Mild forms of HC usually resolve with supportive treatment. However, severe HC may require additional therapies, including hyperbaric oxygen treatment, amifostine, antiviral therapy such as vidarabine or cidofovir, factor VII, bladder irrigation with intravesicular instillation of $\varepsilon$ aminocaproic acid, methyl prednisolone or formalin, cystoscopy and cauterization, and even cystectomy. In addition to acrolein, viral infections such as adenovirus and BK virus (1) have been implicated in the etiology of HC. Therefore therapies directed against viral infections may also be useful in the treatment of HC when appropriate. Further studies are needed to determine the appropriate use of antiviral therapy in virus-associated HC.

Despite the preventative use of mesna, HC is observed in $33 \%$ of the patients treated with IFS. This observation stresses the need for novel therapies as additional prevention for acrolein induced HC. Mota et al (2) investigated the role of recombinant human interleukein-11 (rhIL-11) in preventing experimental IFS-induced HC in Swiss 
mice and have published their results in this issue of the journal. The observations of Mota et $\mathrm{al}^{2}$ demonstrate that rhIL-11 partially prevents IFSinduced HC, presumably due to its antiinflammatory properties and ability to downregulate many pro-inflammatory cytokines. Thus, rhIL-11 may be a very useful adjunct in prevention of HC induced by IFS. These data support that rhIL-11 be used in clinical trials to investigate its role in prevention of $\mathrm{HC}$ in humans.

\section{REFERENCES}

1. Cheerva AC, Raj A, Bertolone SJ, Bertolone K, Silverman CL: BK virus-associated hemorrhagic cystitis in pediatric cancer patients receiving high-dose cyclophosphamide. J Pediatr Hematol Oncol. 2007; 29: 617-21.

2. Mota JM, Brito GA, Loiola RT, Cunha FQ, Ribeiro RA: Interleukin-11 Attenuates Ifosfamide-Induced Hemorrhagic Cystitis. Int Braz J Urol. 2007; 33: 70410.

\author{
Dr. Ashok Raj \\ Dr. Alexandra C. Cheerva \\ Dr. Salvatore J. Bertolone \\ Kosair Children's Hospital \\ Pediatric Hematology \& Oncology \\ University of Louisville \\ Louisville, KY, USA \\ E-mail: acchee01@louisville.edu
}

